\title{
O sistema alimentar e ambientes alimentares na segurança alimentar e nutricional: Um olhar para a realidade moçambicana, distrito de Sussundenga
}

\author{
The food system and food environments in food and nutrition security: A look at the Mozambican \\ reality, Sussundenga district \\ El sistema alimentario y los entornos alimentarios en la seguridad alimentaria y nutricional: Una \\ mirada a la realidad de Mozambique, distrito de Sussundenga
}

\section{Resumo}

O estudo do sistema alimentar não encontra explicação somente na lógica da produção ou de necessidades nutricionais. Neste contexto, este artigo objetivou, descrever os sistemas alimentares, incluindo os ambientes alimentares, buscando compreender suas possíveis influências na segurança alimentar e nutricional (SAN). Trata-se de estudo de caso, de caráter descritivo, de abordagem qualitativa, realizado no distrito de Sussundenga em Moçambique. O delineamento do trabalho foi realizado por meio da observação sistemática com diário de campo e mapa-falante, envolvendo as comunidades de Sussundenga-sede, Munhinga e Chidowa, como base para um modelo explicativo das interações e influências entre as diversas variáveis. Os resultados mostram que o sistema alimentar, nos componentes geofísicos e ambientais, com destaque para as mudanças climáticas, pode ser dos componentes que mais pode afetar a SAN das comunidades, incluindo os ambientes alimentares. Por sua vez, a análise dos ambientes alimentares permitiu constatar que o ambiente nas vias públicas pode apresentar maiores riscos à saúde humana, sobretudo, às crianças, em parte devido a questões ligadas à exposição de alimentos em condições higiênico sanitárias deficientes, suscetíveis de originar doenças que podem causar a desidratação devido a vômitos e diarréias, quando consumidos os alimentos nas condições em que são comercializados. Assim, são necessárias estratégias e ações para a melhoria dos sistemas alimentares, enfatizando a conscientização e a resiliência da população, na melhoria das escolhas alimentares com vista a promoção da saúde e prevenção de doenças, por meio da qualidade nutricional e segurança dos alimentos.

Palavras-chave: Sistemas alimentares; Ambientes alimentares; Segurança alimentar e nutricional.

\begin{abstract}
The study of the food system does not find an explanation only in the logic of production or nutritional needs. In this context, this article aimed to describe food systems, including food environments, seeking to understand their possible influences on food and nutrition security (FNS). This is a case study, of a descriptive character, with a qualitative approach, carried out in the district of Sussundenga in Mozambique. The design of the work was carried out by systematic observation with a field diary and a talking map, involving the communities of Sussundenga-headquarters, Munhinga and Chidowa, as a basis for an explanatory model of the interactions and influences between the various variables. The results show that the food system, in geophysical and environmental components, with emphasis on
\end{abstract}


climate change, may be one of the components that can most affect the FNS of communities, including food environments. In turn, the analysis of food environments has shown that the environment on public roads may present greater risks to human health, especially to children, partly due to issues related to the exposure of food in poor hygienic conditions, susceptible to causing diseases transmitted with symptoms of dehydration due to vomiting and diarrhea, when food is consumed under the conditions in which it is marketed. Thus, strategies and actions are needed to improve food systems, emphasizing the population's awareness and resilience, as well as the empowerment of women to improve food choices with a view to promoting health and preventing diseases, through quality. nutrition and food safety.

Keywords: Food systems; Food environments; Food and nutrition security.

\section{Resumen}

El estudio del sistema alimentario no encuentra solo la lógica de producción o necesidades nutricionales. En este contexto, el artículo tuvo como objetivo describir los sistemas alimentarios, incluidos los entornos alimentarios, buscando comprender sus posibles influencia en la seguridad alimentaria y nutricional (SAN). Se trata de un estudio de caso, de carácter descriptivo, con enfoque cualitativo, realizado en el distrito de Sussundenga en Mozambique. Para ello se utilizó la observación sistemática, la anotación en el diario de campo y el mapa parlante, involucrando a las comunidades de Sussundenga-sedes, Munhinga y Chidowa, a partir de modelos que abordan las interacciones e influencias entre las distintas variables. Los resultados demuestran que el sistema alimentario, en componentes geofísicos y ambientales, con énfasis en el cambio climático, puede ser uno de los componentes que más puede afectar la RAS de las comunidades, incluidos los ambientes alimentarios. A su vez, un análisis de los entornos alimentarios encuentra que el medio ambiente en la vía pública puede presentar mayores riesgos para la salud humana, especialmente para los niños, en parte debido a problemas relacionados con la exposición de alimentos en condiciones higiénicas y sanitarias deficientes, que probablemente causen diarrea. enfermedades, cuando los alimentos se consumen en las condiciones en las que se comercializan. Así, se necesitan estrategias y acciones para mejorar los sistemas alimentarios, enfatizando la conciencia y resiliencia de la población, en la mejora de las opciones alimentarias con miras a promover la salud y prevenir enfermedades, a través de la calidad nutricional y la seguridad alimentaria.

Palabras clave: Sistemas alimentarios; Ambientes alimentarios; Seguridad alimentaria y nutricional.

\section{Introdução}

O sistema alimentar pode ser caracterizado como todos elementos e atividades de alguma forma associados aos alimentos, como a produção, distribuição e consumo dos alimentos, além dos seus resultados ambientais e socioeconômicos (Meybeck \& Gitz, 2017). Por essa razão, tem sido cada vez mais recomendada, por organismos internacionais, a adoção e transformação de sistemas alimentares, como uma das medidas importantes para a materialização dos Objetivos de Desenvolvimento Sustentável (ODS), fundamentalmente em relação ao segundo ODS que visa o combate à fome e a desnutrição em todas as suas formas, assim como a promoção da agricultura sustentável (Painel de Especialistas de Alto Nível [HLPE], 2020).

Neste contexto, após anos de progresso na luta contra a fome, a partir de 2015 o número de pessoas passando fome voltou a aumentar novamente no mundo, representando um enorme desafio para os sistemas alimentares (Organização das Nações Unidas para Agricultura e Alimentação [FAO], 2019). Somando-se a isso, esses sistemas são frequentemente colocados à prova diante de adversidades, como a ocorrência de fenômenos naturais, conflitos armados (FAO, 2019), entre outras, decorrentes de práticas negativas, como a produção de alimentos na base de aditivos químicos ou agrotóxicos, cultivo de monocultura e a criação massiva de animais, que origina doenças que tem afetado as cadeias de abastecimento de alimentos e a segurança alimentar e nutricional (SAN) (HLPE, 2020).

Para evitar que a fome continue aumentando consideravelmente nos próximos tempos, sobretudo, nos países africanos, os sistemas alimentares podem desempenhar um papel importante. Daí a necessidade de sua adaptação, cada vez mais consistente, para lidar com as adversidades (Programa das Nações Unidas para o Meio Ambiente [PNUMA], 2020).

Sabe-se que os sistemas alimentares abrangem não apenas atividades de cadeia alimentar, mas também os resultados dessas atividades e sua governança (Ericksen, 2008; Ingram, 2011). Na prática, todos os seres humanos participam dos sistemas alimentares, por motivos variados, como por exemplo, meios de subsistência (Vermeulen, et al., 2012; Godfray, 
2010).

Estudos realizados em países africanos, envolvendo crianças residentes em lugares com maior acesso ao alimento, apontaram a existência de diferenças significativas no estado nutricional dessas crianças, quando comparadas com as que viviam em locais de menor disponibilidde de alimento e baixa diversidade alimentar, ressaltando, deste modo, a importância dos sistemas alimentares no contexto dos ambientes alimentares, no estado nutricional e na saúde dos indivíduos, em especial das crianças (Ganpule-Rao, et al., 2019),

Dessa maneira, importa destacar que, em Moçambique, embora tenha havido progresso na redução da mortalidade infantil e, apesar dos avanços tecnológicos e sociais, cresce o número de crianças sofrendo as consequências de má alimentação e de um ambiente que parece não considerar as suas necessidades (Granheim, 2013), num contexto em que a desnutrição crônica continua sendo problema nutricional mais importante do país, que afeta quase metade de crianças menores de cinco anos, colocando o país na posição dos que apresentam maiores taxas na África e nos outros continentes. Além disso, o nível de atraso no crescimento moderado a grave das crianças, se situa na ordem de 43\%, no nível nacional, somando-se o fato de o país já ter notificado casos consideráveis de pelagra, uma doença relacionada à deficiência de vitamina B3, resultante da diversidade alimentar limitada (Fundo das Nações Unidas para a Infância [UNICEF], 2016; UNICEF, 2019). A situação revela-se muito mais preocupante nas áreas rurais.

É importante notar que, é nos momentos pós-crise que se oferecem maiores oportunidades para transformação dos sistemas alimentares, tornando-os resilientes a crises futuras. Para que isso aconteça, algumas mudanças têm sido sugeridas pelos organismos das Nações Unidades, algumas das quais relacionadas ao estabelecimento de cadeias de suprimentos alimentares, onde os alimentos produzidos localmente podem ser distribuídos com eficiência, no sentido de ver reduzidos os riscos de insegurança alimentar, de ocorrência da desnutrição, e de aumentos de preços nos principais produtos alimentares. Para tanto, é necessária uma transformação rural com vista à capacitação dos pequenos produtores e integrá-los na economia dos sistemas alimentares, promoção de dietas saudáveis, tornando os alimentos saudáveis mais acessíveis. Na sequência, sugere-se que se promova a agricultura sustentável e regenerativa, com vista a devolver, sobretudo, a saúde dos solos (Pnuma, 2020). Assim, com a promoção de sistemas alimentares mais sensíveis à nutrição e ações concretas visando o empoderamento da mulher (Granheim, 2013), onde questões sobre o cuidado com a família e com a alimentação são devidamente discutidas no sentido de conscientização de que tais responsabilidades não são de responsabilidade exclusiva das mulheres.

Deste modo, considerando o importante papel atribuído aos sistemas alimentares, este trabalho teve como objetivo, descrever o sistema alimentar, buscando compreender suas interações e possíveis influências na segurança alimentar e nutricional, no distrito de Sussundenga, Moçambique.

\section{Metodologia}

Foi conduzido estudo de caso, de abordagem qualitativa (Pereira, et al., 2018), adotando como recurso a observação sistemática por meio de anotações nos diários de campo sobre todos os aspectos relevantes (Marconi \& Lakatos, 2003), e mapa falante considerado eficaz para fazer leitura da realidade (Ferreira \& Pereira, 2013). Foi realizado em três comunidades do distrito de Sussundenga, província de Manica em Moçambique: Sussundenga-Sede, Munhinga e Chidowa, entre os meses de abril e outubro de 2019.

Sussundenga é um distrito localizado na província central de Manica, em Moçambique, onde a maioria da população dedica-se essencialmente à atividade agrícola (Ministério de Administração Estatal [MAE], 2005). Conta com 182.013 habitantes. O distrito está dividido em quatro postos administrativos: Dombe, Mohoa, Rotanda e Sussundenga-sede. É um dos locais tidos como prioritários na estratégia de desenvolvimento nacional (MAE, 2005). Possui escolas que lecionam o ensino primário e secundário. 
Na etapa de observação sistemática, foram realizadas visitas em todas as três comunidades, onde maior atenção foi dada à Sussundenga-Sede, tendo em conta a variedade de ambientes alimentares (doméstico, na via pública, organizacional, de restauração e abastecimento). Igualmente permitiu a sistematização de principais aspectos analisados, durante as visitas às comunidades e estabelecimentos que possibilitaram a aproximação junto das comunidades e pessoas relevantes. Especificamente para compreender as questões relacionadas ao ambiente alimentar organizacional, considerou-se a escola Primária de Munhinga.

A construção do mapa-falante foi coletiva, com base nas percepções dos participantes (43 mulheres e 58 homens), dos quais 10 estiveram envolvidos ativamente no processo de construção na comunidade Chidowa, por tratar-se da mais distante dos estabelecimentos comerciais e do acesso aos serviços sociais básicos. Ao construí-lo, os participantes fizeram uma representação de como vêem a situação do território. Assim, foi possível coletar, entre outros aspectos, as informações referentes à localização dos pontos de venda de produtos alimentícios (feiras-livres). Todos os participantes foram selecionados por critérios de conveniência.

Para a análise de resultados, o estudo baseou-se em Trochim (1989), que aconselha a utilização da lógica, consoante correspondências de padrão de características que examinam as inter-relações entre vários aspectos. Para a sistematização dos dados (sistemas alimentares), utilizou-se o modelo proposto pelo Painel de Especialistas de Alto Nível (2017) e para os ambientes alimentares recorreu-se ao modelo de Gálvez Espinoza et al. (2017).

\section{Resultados e Discussão}

Considerando o papel central do sistema alimentar nos ambientes alimentares e na SAN, passaremos a apresentar as percepções em torno da estrutura do sistema alimentar, a partir do qual também discutem-se as ações prioritárias em Moçambique visando à nutrição e, posteriormente discorre-se em torno dos ambientes alimentares. Neste contexto, na Figura 1 encontra-se apresentado a estrutura dos sistemas alimentares em Sussundenga. 
Figura 1: Estrutura dos sistemas alimentares: tendências e desafios, em Sussundenga, 2019.

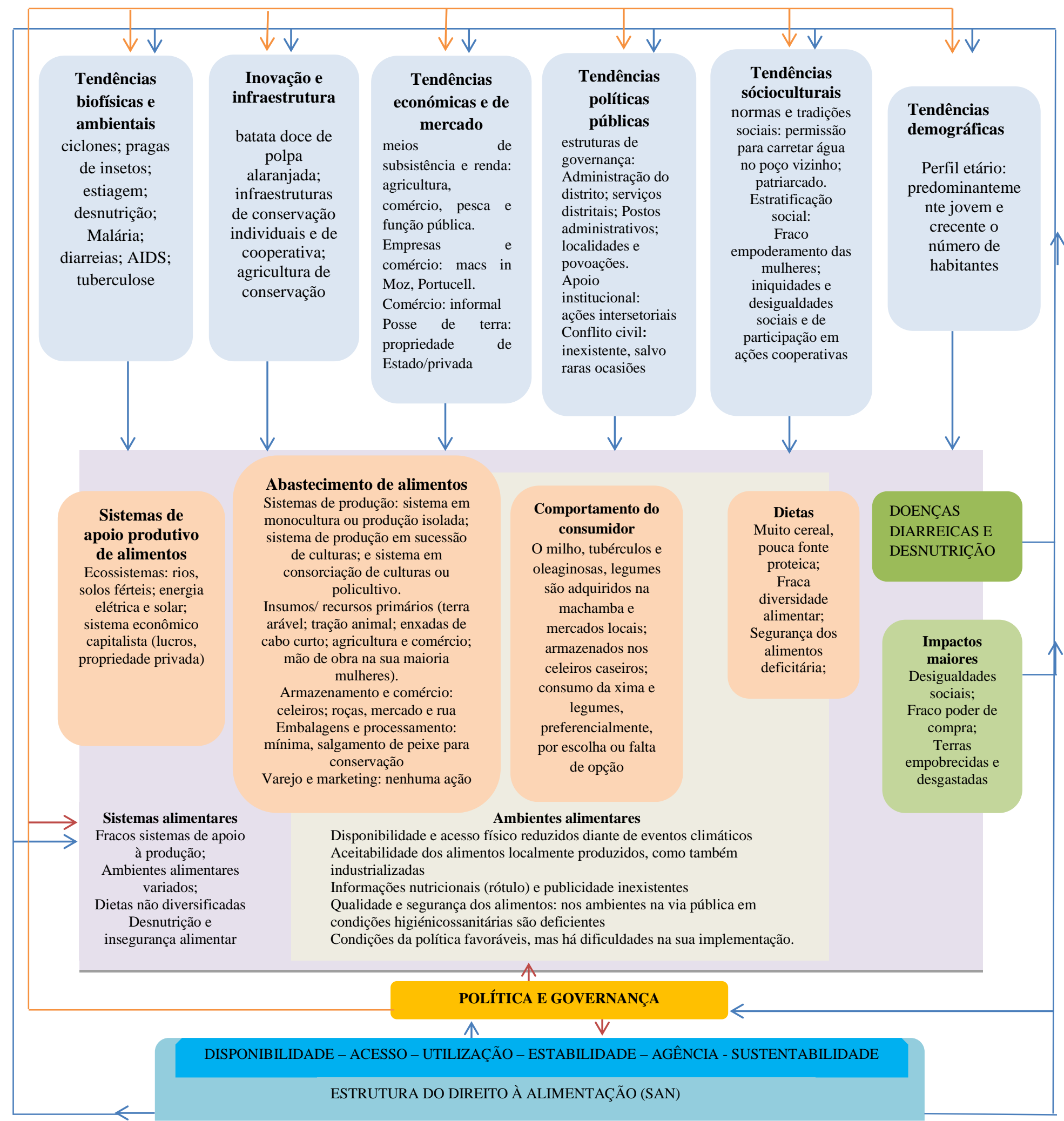

Fonte: Figura adaptada de HLPE (2020).

Na Figura 1 apresenta-se a percepção da estrutura do sistema alimentar do distrito de Sussundenga, baseada nas dimensões propostas pelo Painel de Especialistas de Alto Nível (HLPE, 2020), de forma a facilitar a compreensão das tendências e desafios a partir das interações entre as diferentes variáveis. Dessa maneira, conforme fica evidenciado, o sistema 
alimentar nas suas relações apresenta-se como complexa, desde a produção, o abastecimento de alimentos, os ambientes alimentares, os comportamentos dos consumidores e a diversidade dietética, que se repercute no estado nutricional dos indivíduos. Vale lembrar que, além dessas dimensões o sistema alimentar engloba as atividades como o processamento, a distribuição, preparação e consumo de alimentos, incluindo os resultados dessas atividades num sentido mais amplo, nas esferas socioeconômica, cultural e ambiental. Por isso, há cada vez maior reconhecimento da necessidade de sua abordagem, fundamentalmente devido a sua potencial contribuição na garantia de um direito fundamental, a alimentação (Waage, et al., 2015; HLPE, 2017; Salcedo Fidalgo \& Morales, 2019; Fanzo, et al., 2020; Maestre, et al., 2017).

Nesse sentido, conforme pode-se ilustrar na Figura 1, existem seis grandes fatores moduladores das tendências, os desafios e potenciais oportunidades nos sistemas alimentares, nomeadamente: biofísicos e ambientais; tecnológicos, inovação e infraestruturas; econômicos e de mercado; políticos institucionais; socioculturais e demográficos.

Deste modo, passamos a discorrer em torno dos fatores moduladores, começando pelo biofísico e ambiental, destacando-se as mudanças climáticas, onde os ciclones ocupam um lugar de destaque, dada a sua frequente ocorrência em Moçambique. Na sequência estão as infestações por insetos que afetam os campos produtivos; a estiagem que torna os campos improdutivos; a Malária e diarreias, em parte decorrentes de problemas de saneamento básico que favorecem o desenvolvimento de agentes patôgenos; a tuberculose, muitas vezes associada à AIDS.

Nesse contexto, as mudanças climáticas impactam significativamente na agricultura, afetando a SAN, no que tange a produção e disponibilidade de alimentos, conforme mostrado em publicação anterior no mesmo contexto e território (Buanango, et al., 2021). Efeitos idênticos também podem se fazer sentir quando surgem as pragas e doenças transmitidas por meio de alimentos (FAO, 2019). Isso pode sugerir que os fenômenos naturais podem afetar de forma importante os sistemas alimentares, situação que pode variar de região, sendo que a maior parte dos impactos negativos pode estar concentrada nas regiões tropicais e nos países subdesenvolvidos, onde já existem altas taxas de fome e pobreza. A exemplo, na África subsaariana, da qual Moçambique faz parte, a seca e os ciclones são dos principais fatores contribuintes no aumento da fome e desnutrição-(HLPE, 2020).

Estudos indicam que mais da metade das pessoas vivendo em situação de insegurança alimentar crônica e na condição de desnutrição estão em países que sofrem ou são afetados por algum tipo de catástrofe ou conflito armado (FAO, 2019; Buanango, et al., 2020).

$\mathrm{Na}$ sequência, o segundo fator modulador do sistema alimentar trata-se da tecnologia, inovação e infraestruturas, no qual pode-se destacar a prática de agricultura sustentável (HLPE, 2020). Embora em Moçambique, particularmente em Sussundenga, tem-se incentivado a prática de agricultura de conservação, um sistema agrícola que utiliza um conjunto de técnicas visando a proteção do solo da erosão, a melhoria da fertilidade do solo, aumento da sua rentabilidade, contribuindo assim para a proteção do meio ambiente, melhorando a sustentabilidade (Barbito \& TendayiNyaruwata, 2015), foi observado que não há grandes tecnologias sob domínio dos pequenos produtores em Sussundenga. Entre outros, os insumos agrícolas, sobretudo, os cereais são conservados após à colheita pela maioria das famílias, para a sementeira da campanha agrícola seguinte, enquanto outras famílias preferem adquirir as sementes nos estabelecimentos comerciais locais.

Sabe-se que as tecnologias criam oportunidade para a eficiência, ao mesmo tempo que se levantam críticas, sobretudo, em relação ao melhoramento genêtico de plantas (HLPE, 2020). Em Sussundenga, pode-se destacar a batata-doce de polpa alaranjada, cujo cultivo tem sido incentivado, tanto pelas autoridades administrativas, quanto por organizações não governamentais, dado o fato de ser uma das fontes de vitamina A. Porém essa prática tem colocado em risco a sobrevivência de variedades nativas, o que deveria ser mais bem discutido

No terceiro fator modulador do sistema alimentar, que se refere ao contexto econômico e de mercado, os debates têm sido conduzidos na direção das implicações do mercado de alimentos para a SAN. Nisso, as cadeias de abastecimento 
agroalimentares parecem atuar em benefício das grandes corporações dentro dos sistemas alimentares. Assim, o acesso limitado à terra, recursos e mercados para os pequenos produtores têm sido indicados como elementos centrais que afetam os meios de subsistência no meio rural (HLPE, 2020). Vale lembrar que a venda da terra em Moçambique é ilegal, pois a lei determina que a terra é propriedade do Estado, sendo por isso proibida a sua venda (Moçambique, 1997). Entretanto, apesar dessa proibição, a venda de terra é um "negócio" que acontece com alguma frequência, inclusive, sob influência de determinadas estruturas administrativas de nível local. A realidade de Sussundenga mostra que as famílias estão ficando sem ou com cada vez menores porções de terra, pois sujeitam-se a vender parte dela, sobretudo para as grandes companhias agrícolas locais. É um cenário crítico e insustentável, sobretudo, no contexto rural em Moçambique, onde a agricultura é a principal atividade para a subsistência das famílias.

$\mathrm{Na}$ variavél sociocultural como fator modulador do sistema alimentar, o empoderamento da mulher é necessário, mas ainda representa um desafio na sociedade moçambicana, sobretudo, em Sussundenga, pois a maior parte das mulheres dedicase apenas a atividades domésticas e cuidados da família. Nesse sentido, estudos indicam que a problemática das desigualdades socioeconômicas representa ameaça à coesão social e estabilidade política (FAO, 2019). Ou seja, altas taxas de desigualdades têm sido associadas ao impacto negativo na redução da pobreza (FAO, 2017; Nações Unidas [UN], 2019).

$\mathrm{O}$ acesso desigual aos recursos agrícolas e distribuição desigual de renda estão relacionados à insegurança alimentar $\mathrm{e}$ nutricional, onde as populações rurais praticantes da agricultura de subsistência são especialmente marginalizadas, particularmente as mulheres rurais moçambicanas que enfrentam a pobreza e barreiras em relação ao acesso a ativos produtivos para a agricultura, como a terra, crédito e insumos agrícolas (FAO, 2017; HLPE, 2019). Além disso, as desigualdades sociais também podem afetar a resiliência das comunidades diante dos desastres ambientais (HLPE, 2019).

Vale destacar que, as normas e valores sociais ou culturais (religião, publicidade, gostos, costumes, modos de preparação, o modo em que as refeições têm lugar, se é preparada ou não alguma comida especial, eventualmente em dias festivos, de que forma a comida produzida localmente é valorizada, etc.) influenciam a demanda do consumidor por certos tipos de alimentos (Ericksen, 2008). No entanto, as manifestações da cultura alimentar podem ser afetadas por um ambiente que não favoreça o acesso ao alimento. Em condições muitas vezes de consumo do alimento que se tem, quando tem, sem qualquer oportunidade de escolha.

Por sua vez, o componente demográfico também é pontuado como um dos determinantes na estrutura do sistema alimentar, na medida em que, as mudanças demográficas afetam os padrões de produção e consumo de alimentos. Em Sussundenga, as taxas de crescimento da população tem vindo a aumentar, tendo em conta que em 1997 sua população estimada era de 92.622 habitantes. Presentemente, como foi mencionado, o distrito conta com cerca de 182.013 habitantes (Instituto Nacional de Estatística [INE], 2011; INE, 2017). No entanto, não parece que a oferta de serviços essenciais tenha aumentado na mesma proporção. A questão de saneamento, por exemplo, não parece que esteja sendo tratada com a prioridade que ela merece, ou seja, na dimensão do crescimento populacional. Como resultado disso, as comunidades, sobretudo, as crianças são acometidas por diarreias, em parte devido as deficientes condições higiênico sanitárias e de acesso à água. Ainda relacionado ao componente demográfico, em estudos tem sido apontada a migração como um elemento que afeta as dietas e a nutrição, na medida em que, a emigração para os meios urbanos, supostamente em busca de melhores condições de vida enfraquece a capacidade das comunidades rurais na produção de alimentos, devido a perda de forças de trabalho (HLPE, 2017; Thow, et al., 2016). Sobre esse assunto, importa referir que, a realidade de Sussundenga não mostrou haver essa movimentação de pessoas para os centros urbanos, pelo contrário, deparamos com situações de mulheres que começavam a vida, vindas de outros lugares após separação dos seus cônjuges.

Quanto ao modulador biofísico, destacam-se o abastecimento alimentar, a utilização excessiva de agrotóxicos, queimadas do solo e invasão de animais silvestres nas terras produtivas, dificuldades para deslocamento dos alimentos de um 
lugar para o outro, devido ao fraco acesso ao transporte e condições para o armazenamento, que pode estar relacionado aos aspectos tecnológicos.

Neste contexto, em relação aos insumos/ recursos primários, em Sussundenga, podem-se destacar a terra arável disponível; tração animal; enxadas de cabo curto; agricultura e comércio, onde a mulher constitui a principal mão-de-obra.

O sistema em monocultura ou produção isolada ocorre quando, em uma determinada área, a produção vegetal ou animal se dá de forma isolada em um período específico, geralmente correspondente a um ano agrícola (Hirakuri, et al., 2012; Chiavenato, 2004). Relacionado a esse sistema, em Sussundenga, nalgumas parcelas produtivas tem havido o cultivo do milho intercalado por períodos de pousio, momentos em que as comunidades deixam as terras por algum período, para cultivar nas baixas (lugares próximos às margens de rio). Por sua vez, o sistema de produção em sucessão de culturas era possível encontrar em tempos atrás, onde por vários anos, nalguns campos produtivos predominava a repetição sazonal de uma sequência de espécies no mesmo espaço. No entanto, com o tempo, esse sistema foi sofrendo transformações, conforme foram se retirando do território, seus principais praticantes, essencialmente companhias estrangeiras. No entanto, é o sistema em consorciação de culturas ou policultivo, que pode-se dizer que é o mais adotado, sobretudo, pelos grandes produtores, através de um sistema consorciado entre o feijão e a mandioca, cultivados nas entrelinhas do milho-(Hirakuri, et al., 2012).

Como se pode depreender, vários fatores podem determinar as tendências da estrutura dos sistemas alimentares, que por sua vez incorpora o ambiente alimentar e que, a depender de suas características, favorece ou não a segurança alimentar e nutricional (Figura 2). 
Figura 2: Esquema de ambientes alimentares em Sussundenga: Chidowa, Sussundenga Sede e Munhinga, 2019.

\section{Acesso e consumo}

Preço de alimentos: inacessível para a maioria Promoção de alimentos: Bebidas (refrigerantes), encontrando-se estampadas pelas paredes e muros. Variedade de alimentos: presença regular de cereais, carnes e bebidas. Raras vezes há vegetais, peixe e frutas.

Disponibilidade de opções saudáveis: Pouca

Modo de preparo: cozido, assado e frito.

Informações nutricionais: Não disponível

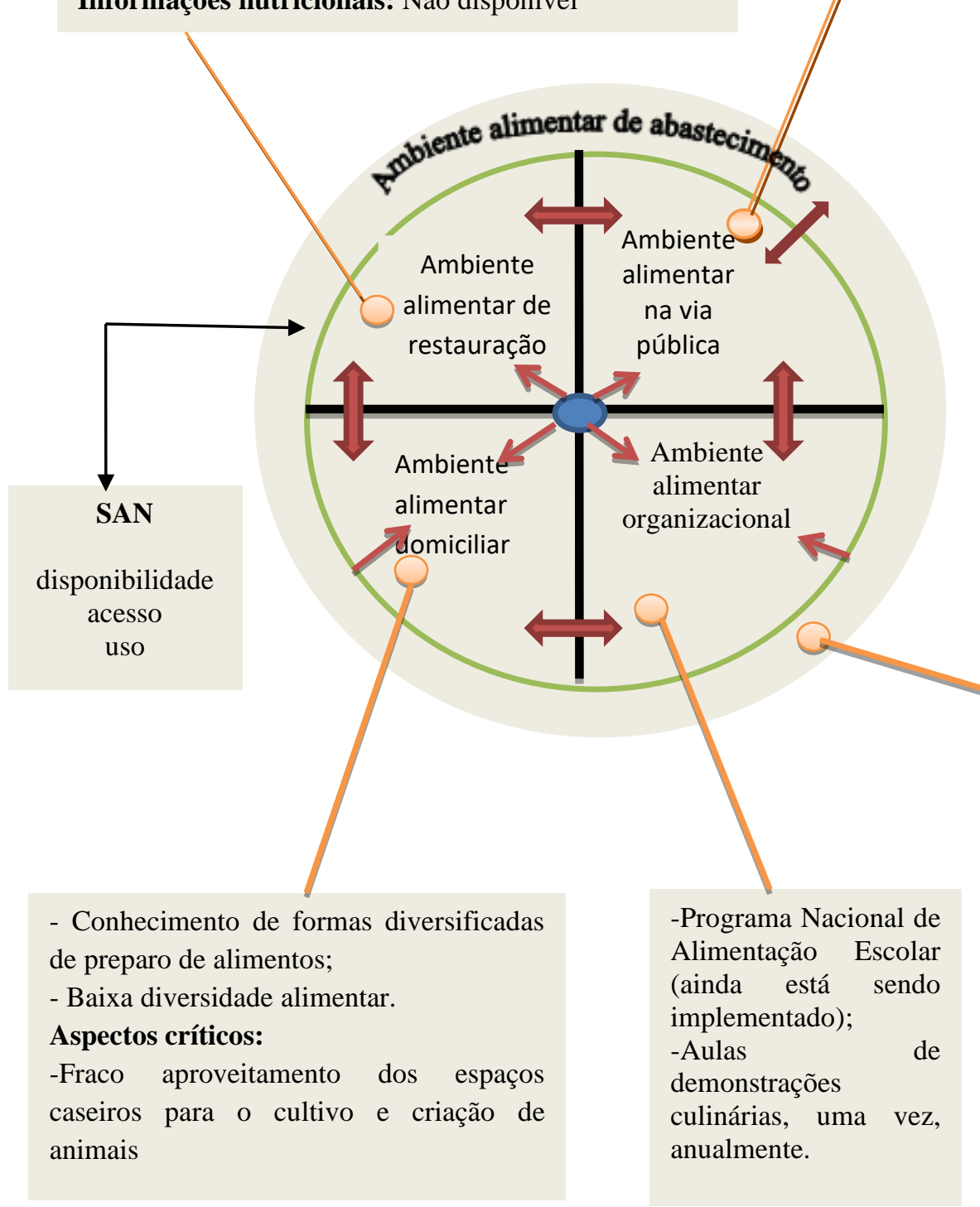

\section{Acesso e consumo}

-Existência de alimentos baratos e de rápido consumo, incluindo os que são preparados na própria via pública; abundância de frutas.

-Venda de alimentos nos transportes de passageiros.

Aspectos críticos: condições higiénicossanitárias deficientes. As crianças correm vários riscos por se fazerem presentes, acompanhadas das respectivas mães.

Fonte: Figura adaptada pelos autores (2021) a partir do modelo de Gálvez Espinosa et al. (2019).

Na Figura 1, apresenta-se a sistematização de ambientes alimentares, com indicação de aspectos que podem condicionar e ou influenciar a SAN.

O ambiente alimentar pode ser descrito como o contexto em que o individuo está inserido, onde as circunstâncias de acesso ao alimento, a disponibilidade, preço e qualidade acabam norteando suas escolhas alimentares (Costa, et al., 2015; Ahmed, 2019). A limitação do acesso aos alimentos saudáveis pode denominar uma área de deserto alimentar devido à pouca densidade de estabelecimentos de aquisição de alimentos in natura (Câmara Interministerial de Segurança Alimentar e 
Nutricional [CAISAN], 2018), ou com o predomínio de estabelecimentos que comercializam alimentos ultraprocessados em relação as opções saudáveis, os pântanos alimentares (Vandevjvere, et al., 2019). Este cenário é sugestionado pela indústria de alimentos, governo, sociedade e por fatores individuais do consumidor de alimentos (Swinburn, et al., 2013)

Vale destacar que, as últimas décadas têm sido caracterizadas pela ocorrência de vários estudos abordando os modelos teóricos conceituais para a compreensão dos ambientes alimentares, seja de forma direta ou indireta (Ahmed, 2019; McKinnon, 2009; Story, et al., 2008; Glanz, et al., 2015; Cummins, 2003). Como vários outros modelos que foram surgindo (Wyse, et al., 2015; Herforth \& Ahmed, 2015), pode-se mencionar o modelo ecológico apresentado por Chan (2015), que evidencia potenciais forças que podem moldar comportamentos de saúde. Apesar disso, ainda são poucos os modelos que tentam explicar e integrar a complexidade de ambientes alimentares e seus fatores condicionantes. Neste contexto, destacamos o modelo de (Gálvez Espinoza, et al., 2017) adotado nesse estudo. Embora tenha sido elaborado num território geográfico diferente, as semelhanças entre as duas realidades e sua abrangência constituíram os principais motivos para a sua escolha. Trata-se de modelo adequado ao estudo de ambiente alimentar e seus fatores condicionantes, pois apresenta categorias e relações que interagem de forma multidirecional e complexa, especulando-se, também, que seja uma das melhores opções quando se pretende ter entendimento sobre a realidade, de forma holística, particularmente em relação à ligação entre os sistemas alimentares, ambientes alimentares e segurança alimentar e nutricional (Gálvez Espinoza, et al., 2017). Na lógica do modelo, existem cinco ambientes alimentares, entre os quais: doméstico ou domiciliar; de restauração; da via pública; de abastecimento e organizacional (Gálvez Espinoza, et al., 2017). Passamos a destacar cada um dos ambientes elencados.

Ambiente domiciliar ou doméstico é tido como um dos mais complexos, devido ao seu nível de produção ou transformação de alimentos. Aliás, sabe-se que é a partir de casa onde marcam-se os primeiros passos para a socialização primária, como também é onde começa e se desenvolve a transmissão da maioria das preferências e tradições alimentares, conforme já evidenciado nalguns estudos (Mennella \& Beauchamp, 2005).

Conforme pode-se notar na Figura 2, um dos aspectos que está influenciando o ambiente alimentar no nível doméstico é a falta de informação para as variadas opções de preparo de alimentos de forma saudável, pois, as mulheres que preparam as refeições, na maioria das vezes, não tendo a possibilidade de se relacionar com as outras pessoas, dada a proibição pelos respectivos parceiros, associada a fatores socioculturais, elas continuam com a mesma forma de preparo, transmitida pela família. Essa falta de informação ou de conhecimento sobre preparo de alimentos é notória, por exemplo no não preparo de suco natural de frutas, num contexto em que o distrito tem muita disponibilidade física de frutas. Devido a isso, a situação tem contribuído para que facilmente as pessoas adiram à compra de produtos industrializados, como bebidas e refrigerantes artificiais (Hartmann \& Siegris, 2013). Somando-se a isso, considerando o fato de as mulheres passarem mais tempo nas ruas ou nas machambas em busca de sustento para a família, sua ausência na casa durante essas atividades pode ter algum impacto no consumo de alimentos para a família, pois, muitas das vezes são crianças menores que acabam tomando a responsabilidade de preparar os alimentos, nessas circunstâncias. Aliás, a esse respeito, Kramer et al. (2012) acreditam que o modo de preparo de alimentos pode influenciar a qualidade e a saúde dos indivíduos, sobretudo, de crianças.

O ambiente alimentar na via pública ou simplesmente "comida de rua", conforme sugerem Bereda et al. (2016), é dos ambientes mais abundantes em Sussundenga. Trata-se de um ambiente relacionado à venda de comida e bebidas prontas ou pré-preparadas, especialmente nas ruas, em torno de centros comerciais ou outros lugares públicos, para o consumo imediato ou sem necessidade de preparo a posterior (Bereda, et al., 2016). A característica comum desse tipo de ambiente é o preço praticado, que é considerado acessível, inclusive por alguns grupos de baixa renda, contribuindo assim para sua maior procura. Vale ressaltar que, para a maioria da população de Sussundenga, depois da agricultura, o comércio informal é uma das principais fontes de renda, como também o é para a maioria da população do país. Apesar de sua importância na economia, os alimentos vendidos nesses lugares apresentam maiores riscos de contaminações, porquanto ficam a céu aberto e, muitas vezes, 
não cobertos (Bereda, et al., 2016), num chão sem asfalto, sofrendo inúmera exposição, sobretudo, durante a noite, pois, enquanto vende-se, funcionários municipais fazem a limpeza, varrendo o chão. Nisso, muita poeira é levantada, tendo em conta o tipo de solo da região, representando um risco à saúde das pessoas que se encontram comercializando, bem como para as pessoas quem por aí passam para comprar os alimentos.

É importante notar que, faz parte da "estratégia" dos vendedores ambulantes nesse ambiente alimentar, levar os alimentos que estão à venda aos seus clientes. Geralmente operam em lugares como terminais de ônibus, escolas, mercado e ruas, sendo as crianças um dos grupos que buscam-se atingir. A situação é preocupante, na medida em que esses locais, também têm a particularidade de atender muito pouco aos requisitos de segurança dos alimentos, representando, deste modo, riscos à saúde, associados à contaminação microbiana (Fórum Global sobre Segurança Alimentar, 2011).

Vários estudos realizados nos países africanos reforçam essa situação sobre os alimentos da rua, devido às condições inapropriadas em que são mantidos, sendo que, além da questão da temperatura, os alimentos já prontos para o consumo são excessivamente manuseados pelas mãos, e comercializados em locais de muita imundície (Bereda et al., 2016). Nesse sentido, aconselha-se que, as Autoridades administrativas direcionem maior atenção na criação de melhores condições sanitárias, acompanhadas de estratégias para a melhoria de conhecimentos sobre o padrão de qualidade da comida.

Dados os constrangimentos apresentados, sobretudo, de ordem sanitária, a atividade de venda de comida na rua é carregada de polêmica. Vale lembrar que, em muitos desses lugares, encontram-se presentes alguns dos principais traços da cultura alimentar da região, e a cultura no geral, onde por exemplo, pode-se ver evidenciada a problemática da relação de gênero, onde além de mulheres, também encontram-se as crianças comercializando (Bereda, et al., 2016).

Passemos ao ambiente alimentar organizacional que refere-se ao local de venda e fornecimento de alimentos nos espaços da organização (Gálvez Espinoza, et al., 2017). No caso em concreto, vale ressaltar que para esse estudo, nos centramos no ambiente alimentar da escola de Munhinga, incluindo o seu entorno. Deste modo, pesquisadores têm cada vez mais reconhecido o papel da alimentação na promoção da saúde e proteção contra doenças. E a escola tem sido apontada como espaço privilegiado para a implementação de ações de promoção da saúde, esperando-se que venha desempenhar papel importante na formação de valores, hábitos, estilos de vida, entre eles, a alimentação, tendo como base práticas alimentares promotoras de saúde (Accioly, 2009).

Constatou-se na escola, a presença de muitos alimentos processados e açucarados. Vale ressaltar que, em Moçambique, embora tenha sido aprovado em 2013 o Programa de Alimentação Escolar (Granheim, 2013), ainda não foi implementado conforme o previsto na escala nacional. Daí que, os alunos da Escola de Munhinga vão com a merenda para à escola, muitas vezes a merenda é mesmo a comida que sobrou de refeições do dia anterior, ou quando isso não acontece, os pais dão dinheiro para que comprem os alimentos disponíveis no recinto ou entorno da escola.

Cabe destacar que, durante a coleta de dados, constituiu oportunidade o decurso de aula sobre demonstrações culinárias na escola, que tem acontecido pelo menos uma vez em cada ano letivo, envolvendo alunos da primeira à sétima séries. Trata-se de um momento em que os alunos levam à escola produtos alimentares para posterior confecionamento, em espaço aberto. A partir da observação dessa atividade, foi notória a preferência por alimentos como macarrão e peixe frito. Nessa ocasião, a professora que orientava a aula preparou um rato frito (conhecido localmente por rato da roça, bastante apreciado e consumido pelas comunidades). Refira-se que, nessa região existe muita disponibilidade de alimentos como vegetais, tubérculos, leguminosas e frutas. Eventualmente nessas ocasiões fosse importante a presença de mais alimentos localmente disponíveis, podendo assim ser um verdadeiro veículo na promoção pela alimentação saudável.

Passemos de seguida ao ambiente de restauração, que engloba, entre outros componentes, restaurantes, locais de preparo rápido de comida, bares e meios de transporte (De Rezende, 2012). As pessoas que acorrem a esses locais, geralmente são adultas e trabalhadoras e, dependendo das circunstâncias, há crianças que também se fazem presentes acompanhadas pelas 
pessoas adultas.

Dos locais de comercialização de refeições observados, dois deles dedicam-se ao preparo de xima (semelhante a polenta, porém mais endurecida), arroz, frango e carne de cabrito; o terceiro estabelecimento prepara arroz, xima, batata-frita, frango assado e cozido, carne bovina, peixe assado e, por vezes, salada de alface, além de hamburguers. O quarto e último estabelecimento, uma lanchonete dedica-se somente ao preparo de hamburguers e sandes de ovo. Vale lembrar que, nessa região, há muita disponibilidade de hortaliças, como couve, alface, repolho, tomate, etc, que podem ser encontrados muito facilmente. Mas o que acontece é que a alface, por exemplo é servida em apenas um dos estabelecimentos e, somente quando solicitada pelo cliente. No seu cardápio os preços de comida são considerados mais elevados quando comparado aos outros, pois chega a equiparar-se aos preços praticados nos centros urbanos.

Todavia, o aspecto comum nesses quatro estabelecimentos de preparo e venda de alimentos é a grande presença de refrigerantes e sucos artificiais de vários tipos, impulsionados pela influência do mercado publicitário. Sabe-se que sucos artificiais, bem como refrigerantes contém aditivos químicos, além de apresentar preocupação com alto teor de açúcar (Toloni, et al., 2011). Com a abundância de frutas na região, como a laranja, banana, abacate, tangerina, manga, e cana-de-açúcar, esperava-se encontrar estabelecimentos preparando suco natural. Além disso, nenhum dos locais apresentava informação nutricional dos pratos oferecidos. A esse respeito, Sloan e Bell (1999) acreditam que seja uma prática comum, sobretudo, nos países africanos, e aconselham que consumidores tenham acesso às informações nutricionais, porquanto aumentaria seu conhecimento sobre nutrição, para que se busca por dietas mais benéficas à saúde, no caso das pessoas que tenham condições para o efeito.

Passemos ao ambiente de abastecimento, que apresenta uma dupla dimensão, pois além de ser um ambiente alimentar por si só, também interfere nos demais ambientes alimentares, e na SAN de forma direta, sobretudo, na disponibilidade e acesso ao alimento (Pessoa, et al., 2015; Chen \& Kwan, 2015). Neste contexto, nas regiões estudadas, o abastecimento tem sido influenciado por determinantes como o acesso ao transporte. A exemplo, o peixe cultivado em cativeiro pelos produtores locais, raras vezes encontra-se disponível nos mercados locais, pois os criadores têm dificuldades de fazer chegar a esses mercados, devido a dificuldades de acesso ao transporte para os centros comerciais, além de dificuldades relacionadas à conservação, tendo por isso, nalgumas vezes como alternativa, recorrido ao processamento mínimo, especificamente ao salgamento e secagem do peixe, um processo que, eventualmente altera a composição nutricional do alimento (Erickson, 2008).

Na perspectiva de compreender o funcionamento dos mercados locais, passamos a destacar a sua organização, com vista a descrever todo o contexto relacionado à venda de alimentos no ambiente na via pública, iniciando pelo maior mercado, trata-se do mercado de Sussundenga-Sede. Concentra em seu interior vários pequenos estabelecimentos comerciais, com diversificados tipos de alimentos: Segundo relato é onde existem infra-estruturas comerciais feitas ainda durante o regime colonial, localmente designadas por bazar. São comercializados neste mercado vários alimentos, entre eles, vegetais, legumes, frutas, leguminosas, tubérculos, cereais. O inhame, a mandioca, a batata-doce e o milho estão entre os alimentos que são preparados no local para a venda já pronto para o consumo.

Cabe ressaltar que, nem todas as pessoas que pretendem comercializar conseguem ter espaço ou recurso para construir seu estabelecimento dentro do bazar, por isso, como alternativa, muitas delas se posicionam nas laterais de estradas, arredores do mercado, para venderem seus produtos alimentares em barracas desmontáveis, feitas de madeira. O número de pessoas varia muito, dependendo dos dias, locais e horários. Nota-se maior movimentação durante os finais de semana, a partir do período da tarde, quando o sol abranda, o número aumenta significativamente, chegando até a atingir centenas de barracas ou pequenos quiosques durante o período da noite, ocupando todos os espaços nas ruas. As crianças são atribuídas a tarefa de venda de refrigerantes, bolachas, ovos cozidos, amendoim, no terminal de transportes, por alegadamente disporem de agilidade 
para correr ao encontro de clientes nos transportes semi coletivos de passageiros.

Nesse mesmo mercado, especificamente, os alimentos processados ou industrializados são comercializados em pequenas lojas pertencentes, na sua maioria, aos cidadãos de origem asiática (bangladesa).

Por sua vez, o mercado de Munhinga, também caracteriza-se pela divisão em pequenos estabelecimentos particulares. Em jeito de apoio, de acordo com relato, as autoridades disponibilizaram espaços para que fossem construídas algumas pequenas lojas, na base de material convencional, porém, a iniciativa não surtiu os efeitos desejados. À semelhança do de Sussundenga-Sede, os espaços à beira de ruas são transformados em locais de comércio informal, onde é possível verificar vários produtos alimentares colocados sobre o chão. Porém, diferentemente do mercado de Sussundenga-Sede, o comércio em Munhinga acontece essencialmente durante o dia, caracterizado por vendas de bolos fritos (bolinhos de chuva), cana-deaçúcar, batata-doce, tangerina, laranja, repolho e peixe. O comércio de produtos alimentares processados e industrializados, na sua maioria, também é feito na sua maioria por estrangeiros.

No interior dos mercados, tanto no de Munhinga quanto no de Sussundenga-Sede, vendem-se diversos tipos de hortaliças, além de peixe-seco e óleo de cozinha (utilizado e descartado). Os comerciantes têm acesso a esse óleo e disponibilizam para a venda a preços mais baixos que o óleo não utilizado. Porém, o óleo usado parece representar maiores riscos à saúde humana, até porque para a sua reutilização, os usuários costumam esquentá-lo, para derretê-lo, o que pode ser um dos indícios de possíveis malefícios que possa causar no organismo humano.

Por seu turno, em Chidowa, os pequenos espaços construídos com recurso ao material local (estacas, bambús, sacos, argila) são transformados em feiras livres. Com a passagem do ciclone Idai em março de 2019, várias barracas foram destruídas, além de ter resultado na redução da disponibilidade de alimentos, sobretudo, mandioca, batata-doce, inhame e milho. Esse fato contribuiu para o aumento dos preços praticados para esses produtos, bem como na redução no seu consumo. Nas barracas que resistiram, ficaram disponíveis apenas produtos industrializados, como o suco artificial em pó, bolachas e caldo Knorr. Em parte, essa pouca disponibilidade e diversidade de alimentos se devem, também, ao fato de não existirem serviços essenciais, aliado ao fraco poder de compra da população. Aliás, sobre isso, Pessoa (2013) indicou no seu estudo que, os estabelecimentos de venda de alimentos estão presentes consoante a renda das pessoas circunvizinhas, o que não é o caso das comunidades de Chidowa. Ou seja, onde há maior renda, também existe um potencial de existência de inúmeros estabelecimentos comerciais (Pessoa, 2013), o que é importante na estratégia de promoção da saúde (Costa, et al., 2015).

Face a essas dificuldades e outras como a questão do acesso à água, a comunidade de Chidowa quando necessita de alimentos como peixe, frango, leguminosas e algumas hortaliças, sujeita-se a percorrer à pé distâncias superiores à $40 \mathrm{~km}$, inclusive para ter acesso aos cuidados básicos de Saúde. Essa situação pode estar na origem de altas taxas de abandono ao tratamento hospitalar e consultas pré-natais, além de interrupção no seguimento do tratamento de desnutrição aguda em crianças.

\section{Considerações Finais}

A discussão feita neste artigo não pretendeu encerrar o debate sobre o tema, mas foi uma tentativa de evidenciar o papel importante e determinante do sistemas alimentar nos ambientes alimentares e na segurança alimentar e nutricional. $\mathrm{O}$ distrito de Sussundenga é somente um recorte destes cenários. Contudo, pode ser que nos diferentes distritos e províncias de Moçambique a situação seja a mesma, de condições de insegurança alimentar e nutricional, face as interações com os seus determinantes ou componentes.

De modo geral, os resultados mostram a importância do sistema alimentar nos ambientes alimentares, bem como sua relevância na segurança alimentar e nutricional. Neste contexto, pode-se dizer que, o sistema alimentar no distrito de Sussundenga apresenta alguns desafios importantes, sobretudo, na questão geofísica e ambiental que afeta a produção, 
disponibilidade, acesso e consumo de alimentos, sobretudo, os ambientes alimentares. As vendas que são efetuadas na via pública, pelas condições higiênicossanitárias associadas a problemas de acesso à água, representa risco para a saúde dos consumidores, sobretudo, para as crianças menores, que ao consumirem os alimentos contaminados, têm maiores riscos de apresentarem consequências mais graves, por meio de doenças transmitidas por alimentos que causam desidratação, em consequência de vómitos e diarréia, podendo afetar a saúde e seu desenvolvimento, inclusive, seu estado nutricional. Enquanto no ambiente alimentar de restauração existe pouca diversidade de alimentos localmente disponíveis.

Os estabelecimentos comerciais podem ser importantes promotores de alimentação saudável, sendo por isso aconselhável que os tomadores de decisões a nível de políticas públicas possam criar condições e sugerir que os proprietários desses estabelecimentos possam melhorar as condições de venda e a diversificação de dietas, contemplando sucos naturais, frutas, hortaliças, leguminosas. Sugere-se ainda que, na escola, as ações relacionadas à alimentação devam valorizar os produtos locais.

Por sua vez, o sistema alimentar e seus vários determinantes ou componentes na sua interação pode estar afetando a SAN, propiciando, assim, maiores riscos para a saúde, em particular, para a condição da desnutrição infantil. A exemplo, fenômenos naturais podem estar reduzindo a disponibilidade de alimentos, piorando a situação nos lugares que já deparam com dificuldades de abastecimento, dado acesso deficitário ao transporte, assim como as vias e limitada capacidade de renda. Somando-se a isso, estão as distâncias para obtenção de alimentos variados e saudáveis nalgumas regiões, aliado aos custos, considerados elevados para a capacidade aquisitiva da maioria nas comunidades.

Para trabalhos futuros, sugere-se a abrangência do lócus da pesquisa e abordagem multidisciplinar, para que mais pesquisadores possam denunciar e alertar sobre os riscos relacionados à manutenção de ambientes e sistemas alimentares deficitários, para que se promovam ambientes capazes de promover a saúde no geral, bem como a SAN, e através disso, poderse reduzir e prevenir a ocorrência da desnutrição nos territórios.

\section{Agradecimentos}

O presente trabalho foi realizado com apoio da Coordenação de Aperfeiçoamento de Pessoal de Nível Superior Brasil (CAPES) - código de financiamento 001.

\section{Referências}

Accioly, E. (2009). A escola como promotora da alimentação saudável. Ciência em Tela, 2(2), 1-9. http://www.cienciaemtela.nu tes.ufrj.br/artigos/0209accioly.pdf.

Ahmed, S., Shanks, C. B., Dupuis, V. \& Pierre, M. (2019). Advancing healthy and sustainable food environments: The Flathead Reservation case study. UNSCN Nutr, 44, 38-45.

Barbito, A., TendayiNyaruwata, L. (2015) A agricultura de conservação e a sua sustentabilidade social. Revista Electrónica de Investigação e Desenvolvimento, 4, 1-15. http://reid.ucm.ac.mz/index.php/reid/article/view/52/67.

Bereda, T. W., Emerie, Y. M., Reta, M. A., \& Asfaw, H. S. (2016). Microbiological safety of street vended foods in Jigjiga City, Eastern Ethiopia. Ethiop J Health Sci, 26, 161-70.

Buanango, M. A., Chume, P. F. C., Oliveira, M. R. M. de. (2021). Efeitos do ciclone Idai na produção, disponibilidade e acesso aos alimentos no distrito de Sussundenga em Moçambique. Research, Society and Development, 10(3), 1-17. 10.33448/rsd-v10i3.13778. -

Buanango, M. A., Ferreira, V. A. D. S., Oliveira, M. R. M. de. (2020). Novo coronavírus SARS-CoV-2 e o agravamento da insegurança alimentar em países africanos com histórico de eventos climáticos e de conflitos armados. Rev. Simbio-Logias, 12(16), 118-141.

Câmara Interministerial de Segurança Alimentar e Nutricional. (2018). Estudo Técnico Mapeamento dos Desertos Alimentares no Brasil. Secretaria-Executiva da Câmara Interministerial de Segurança Alimentar e Nutricional Ministério do Desenvolvimento Social. https://aplicacoes.mds.gov.br/sagirmps/noticias/arquivos/files/Estudo_tecnico_mapeamento_desertos_alimentares.pdf.)

Chan, C. (2015). Food Environment, Health and Chronic Disease. Green paper prepared for the Alberta Institute of Agrologists. http://www.albertaagrologists.ca/document/1911/Mar30_Green\%20PaperFinal.pdf. 
Chen, X. \& Kwan, M-P. (2015). Contextual uncertainties, human mobility, and perceived food environment: The uncertain geographic context problem in food access research. Am J Public Health, 105(9), 1734-1737. 10.2105/AJPH.2015.302792.

Chiavenato, I. (2004). Introdução à teoria geral da administração. Editora Campus, (7a ed.).

Costa B. V. de L., Oliveira, C. D. L. \& Lopes, A. C. S. (2015). Ambiente alimentar de frutas e hortaliças no território do Programa da Academia da Saúde. Cadernos de Saúde Pública, 31 (S), 1-11. http://dx.doi.org/10.1590/0102-311X00027114.

Cummins, S. C. J. (2003). The local food environment and health: Some reflections from the United Kingdom. Am J Public Health, 93(4), 521. 10.2105/ajph.93.4.521.

De Rezende, D. C., De Avelar, A. E. S. (2012). Factors that influence the consumption of food outside the home in Brazil. Int J Consum Stud, 36(3), 300-306. https://doi.org/10.1111/j.1470-6431.2011.01032.

Downs, S. M., Ahmed, S., Fanzo, J., \& Herfort, H. (2020). Food Environment Typology: Advancing an Expanded Definition, Framework, and Methodological Approach for Improved Characterization of Wild, Cultivated, and Built Food Environments toward Sustainable Diets. Foods, 9 (4), 2-32. doi:10.3390/foods 9040532 .

Ericksen, P. J. (2008). Conceptualizing food systems for global environmental change research. Global Environmental Change, 18, 234-245. doi:10.1016/j.gloenvcha.2007.09.002.

Fanzo, J., Haddad, L., McLaren, R. Marshall, Q., Davis, C., Herforth, A., Jones, A., Beal,T., Tschirley, D., Bellows, A., Miachon, L., Gu, Y., Bloem, M., \& Kapuria, A. (2020). The Food Systems Dashboard is a new tool to inform better food policy. Nature Food, 1, 243-246.

Ferreira, A. L., Pereira, M. F. W. (2013). O Mapa Falante como Instrumento do Processo Ensino-Aprendizado do Aluno de Medicina: Relato de Experiência. Revista de Pediatria SOPERJ, 14(1), 29-32.

Fórum Global sobre Segurança Alimentar e Nutrição (2011). Alimentos de venta callejera: el camino a seguir para una mejor seguridad alimentaria y nutrición. http://www.fao.org/fsnforum/sites/default/files/file/73_street_foods/summary_73_street_food_sp.pdf.

Fundo das Nações Unidas para a Infância. (2019). A má alimentação prejudica a saúde das crianças em todo o mundo, adverte o UNICEF. A pobreza, a urbanização, as mudanças climáticas e as más escolhas alimentares resultam em dietas prejudiciais para a saúde. https://www.unicef.org/mozambique/comunicados-de-imprensa/m\%C3\%A1-alimenta\%C3\%A7\%C3\%A3o-prejudica-sa\%C3\%BAde-das-crian\%C3\%A7asem-todo-o-mundo-adverte-o.

Fundo das Nações Unidas para a Infância. (2016). Nutrição. Para cada criança, nutrição. https://www.unicef.org/mozambique/nutri\%C3\%A7\%C3\%A3o.

Ganpule-Rao, A. V., Roy, D., Karandikar, B. A., Yajnik, C. S., \& Rush, E. C. (2019). Food Access and Nutritional Status of Rural Adolescents in India: Pune Maternal Nutrition Study. American Journal of Preventive Medicine, 58(5), 728-735. 10.1016/j.amepre.2019.10.017.

Gálvez Espinoza, P., Egaña, D., Masferrer, D. \& Cerda, R. (2017). Propuesta de un modelo conceptual para el estudio de los ambientes alimentarios en Chile. Rev Panam Salud Publica, 41, 1-9. 10.26633/ RPSP.2017.169.

Glanz, K., Sallis, F., Saelens, B. E., Frank, L. D. (2005). Critical Issues and Trends Healthy Nutrition Environments: Concepts and Measures. American Journal of Health Promotion, 19(5), 330-333. 10.4278/0890-1171-19.5.330.

Godfray, H. C. J., Beddington, J. R., Crute, I. R., Haddad, L., Lawrence D., Muir, J. F., Pretty, J., Robinson, S., Thomas, S. M., Toulmin, C. (2010). Food security: the challenge of feeding 9 billion people. Science, $327,812-818$.

Granheim, C. I. O. (2013). Análise de Políticas Nacionais Impacto dos sistemas agrícolas e alimentares na Nutrição Moçambique. United Nation Systems, Standig commitee of nutrition.

Hartmann, C., Dohle, S., \& Siegrist, M. (2013). Importance of cooking skills for balanced food choices. Appetite, 65, 125-131. https://doi.org/10.1016/j.appet.2013.01.016.

Herforth, A., \& Ahmed, S. (2015). The food environment, its effects on dietary consumption, and potential for measurement within agriculture-nutrition interventions. Food Security, 7(3), 505-520. 10.1007/s12571-015-0455-8.

Hirakuri, M. H., Debiasi, H., Procópio, S. de. O., Franchini, J. C., Castro, C.de. (2012). Sistemas de Produção: conceitos e definições no contexto agrícola. Londrina: Empresa Brasileira de Pesquisa Agropecuária Embrapa Soja, https://ainfo.cnptia.embrapa.br/digital/bitstream/item/69333/1/Doc-335-OL.pdf

Ingram J. (2011). A food systems approach to researching food security and its interactions with global environmental change. Food Secur. 3, 417-431

Instituto Nacional de Estatística. (2011). Estatística do Distrito de Sussundenga. http://www.ine.gov.mz/estatisticas/estatisticas-territoriasdistritais/manica/2011/distrito-de-sussundenga.pdf/view.

Instituto Nacional de Estatística. (2017). Censo 2017 Brochura dos Resultados Definitivos do IV RGPH - Nacional. http://www.ine.gov.mz/iv-rgph2017/mocambique/censo-2017-brochura-dos-resultados-definitivos-do-iv-rgph-nacional.pdf/view

Kramer, R. F., Coutinho, A. J., Vaeth, E., Christiansen, K., Suratkar, S., \& Gittelsohn, J. (2012). Healthier home food preparation methods and youth and caregiver psychosocial factors are associated with lower BMI in African American youth. J Nutr, 142(5), 948-954. https://doi.org/ 10.3945/jn.111.156380.

Maestre, M.., Poole, N. \& Henson, S. (2017). Assessing food value chain pathways, linkages and impacts for better nutrition of vulnerable groups. Food Policy, 68, 31-39.

Marconi, M. de A., \& Lakatos, E. M. (2003). Fundamentos de metodologia científica. (5a ed.), Atlas 
McKinnon, R. A., Reedy, J., Morrissette, M. A., Lytle, L. A., \& Yaroch, L. A. (2009). Measures of the Food Environment A Compilation of the Literature, 1990-2007. American Journal of Preventive Medicine, 36, 124-133. doi:10.1016/j.amepre.2009.01.012.

Mennella, J. A., \& Beauchamp, G. K. (2005). Understanding the origin of flavor preferences. Chem Senses, 30(S1), 242-343. 10.1093/chemse/bjh204.

Meybeck, A. \& Gitz, V. (2017). Sustainable diets within sustainable food systems. Proc Nutr Soc. England, 76(1), 1-11.

Ministério de Administração Estatal. (2005). Perfil do distrito de Sussundenga província de Manica. Maputo.

Moçambique. (1997). Lei de Terras, Lei $n^{o} 19 / 97$ de 1 de Outubro. https://www.sheltercluster.org/sites/default/files/docs/lei_terras_mocambique.pdf

Nações Unidas. (2019). Special edition: progress towards the Sustainable Development Goals: Report of the Secretary-General. Rome. https://undocs.org/E/2019/68.

Organização das Nações Unidas para Agricultura e Alimentação. (2019). The State of Food and Agriculture 2019. Moving forward on food loss and waste reduction. Rome. http://www.fao.org/3/ca6030en/ca6030en.pdf.

Organização das Nações Unidas para Agricultura e Alimentação. (2017). The Future of Food and Agriculture - Trends and Challenges. Rome.

Painel de Especialistas de Alto Nível. (2020). Food security and nutrition: building a global narrative towards 2030. A report by the High Level Panel of Experts on Food Security and Nutrition of the Committee on World Food Security, Rome.

Painel de Especialistas de Alto Nível. (2019). Agroecological and other innovative approaches for sustainable agriculture and food systems that enhance food security and nutrition. A report by the High Level Panel of Experts on Food Security and Nutrition of the Committee on World Food Security. HLPE report 14. Rome. http://www.fao.org/3/ca5602en/ca5602en.pdf.

Painel de Especialistas de Alto Nível. (2017). Nutrition and food systems. A report by the High Level Panel of Experts on Food Security and Nutrition of the Committee on World Food Security, Rome. http://www.fao.org/3/i7846e/i7846e.pdf.

Painel de Especialistas de Alto Nível. (2013). Biofuels and food security. A report by the High Level Panel of Experts on Food Security and Nutrition of the Committee on World Food Security. HLPE report 5. Rome. http://www.fao.org/3/a-i2952e.pdf.

Pereira, A. S., Shitsuka, D. M., Parreira, F. J., \& Shitsuka, R. (2018). Metodologia da pesquisa científica. UFSM. https://www.ufsm.br/app/uploads/sites/358/2019/02/Metodologia-da-Pesquisa-Cientifica_final.pdf.

Pessoa, M. C. (2013). Ambiente alimentar e consumo de frutas, legumes e verduras em adultos de Belo Horizonte - MG. [Tese Doutorado, Universidade Federal de Minas Gerais].

Pessoa, M. C., Mendes, L. L., Gomes, C. S., Martins, P. A. \& Velasquez-Melendez, G. (2015). Food environment and fruit and vegetable intake in a urban population: A multilevel analysis. BMC Public Health. 15(1), 1-8. doi: 10.1186/s12889-015-2277-1.

Programa das Nações Unidas para o Meio Ambiente. (2020). Por que precisamos de sistemas alimentares sustentáveis no mundo pós-pandemia. https://www.unep.org/pt-br/noticias-e-reportagens/reportagem/por-que-precisamos-de-sistemas-alimentares-sustentaveis-no-mundo.

Salcedo Fidalgo, H. \& Morales, J. C. (2019). Nutritional Assessment Methodologies: Challenges and Opportunities for the Full Realization of the right to food and Nutrition. Frontiers in Nutrition, 6, 1-4. https://doi.org/10.3389/fnut.2019.00035.

Sloan, M. E., \& Bell, L. N. (1999). Fat content of restaurant meals: comparison between menu and experimental values. Journal of the American Dietetic Association, 99(6), 731-733.

Story, M., Kaphingst, K. M., Robinson-O’Brien, R., Glanz, K. (2008). Creating healthy food and eating environments: Policy and environmental approaches. Annu. Rev. Public Health, 29, 253-272.

Swinburn, B., Sacks, G., Vandevijvere, S., Kumanyika, S., Lobstein, T., Neal, B. et al. (2013). INFORMAS (International Network for Food and Obesity/noncommunicable diseases Research, Monitoring and Action Support): overview and key principles. Obes Rev., 14(Suppl 1), 1-12.

Thow, A. M., Fanzo, J. \& Negin, J. (2016). A systematic review of the effect of remittances on diet and nutrition. Food and Nutrition Bulletin, $37(1)$ : 42-64.

Toloni, M. H. De. A., Longo-Silva, G., Goulart, R. M. M., \& Taddei, J. A. de. A. C. (2011). Introdução de alimentos industrializados e de alimentos de uso tradicional na dieta de crianças de creches públicas no município de São Paulo. Revista de Nutrição, 24(1), 61-70. https://doi.org/10.1590/S141552732011000100006 .

Trochim, W. (1989). Outcome pattem matching and program theory. Evaluation and Program Planning, 12(4), 355-366. https://doi.org/10.1016/01497189(89)90052-9.

Vandevijvere, S., Mackay, S., D’Souza, E., Swinburn, B. (2019). The first INFORMAS national food environments and policies survey in New Zealand: A blueprint country profile for measuring progress on creating healthy food environments. Obes Rev., 20(2), 141-160.

Vermeulen, S. J., Campbell, B. M., \& Ingram, J. S. I. (2012). Climate Change and Food Systems. Annu. Rev. Environ. Resour. 37, 195-222. 0.1146/annurevenviron-020411-130608.

Waage, J., Yap, C., Bell, S., Levy, C. \& Mace, G., Pegram, T., Unterhalter, E., Dasandi, N., Hudson, D., Kock, R.., Mayhew, S., Marx, C., Poole, N. (2015). Governing the UN Sustainable Development Goals: Interactions, infrastructures, and institutions. The Lancet Global Health, 3(5), 251-252. 10.1016/S2214$109 \mathrm{X}(15) 70112-9$. 
Research, Society and Development, v. 10, n. 6, e31810615774, 2021

(CC BY 4.0) | ISSN 2525-3409 | DOI: http://dx.doi.org/10.33448/rsd-v10i6.15774

Wyse, R., Wolfenden, L., \& Bisquera, A. (2015). Characteristics of the home food environment that mediate immediate and sustained increases in child fruit and vegetable consumption: mediation analysis from the Healthy Habits cluster randomised controlled trial. Int J Behav Nutr Phys Act, 12(1), 2-9. https://doi.org/: 10.1186/s12966-015-0281-6. 